\title{
Experimentelle Untersuchungen über den intermediären Stoffwechsel im Muskel bei Fieber.
}

\author{
I. Mitteilung. \\ Gas- und Kohlehydratstoffwechsel im Muskel bei \\ experimentellem Fieber.
}

Von

Kesae Adachi.

(安達今朝副)

(Aus der Medizinischen Klinik von Prof. Dr. T. Kato, Kaiserliche Universität zu Sendai.)

Es gibt nur wenige Untersuchungen über den Milchsäurestoffwechsel bei Fieber, deren Ergebnisse noch nicht übereinstimmen. Fries ${ }^{1)}$ hat aus seinen Versuchen betreffs der Pneumonie und einiger anderer fieberhafter Krankheiten festgestellt, dass die Blutmilchsäure auch bei Fieber nicht zunimmt. Hingegen hat Margret $\mathrm{h}^{233)}$ beobachtet, dass die Blutmilchsäure des Abdominaltyphuskranken, des Phthisikers und des Pneumoniekranken bei schweren Fällen zunimmt. Ebenfalls haben Osato, Oba u. Kasai ${ }^{4)}$ Zunahme der Blutmilchsäure bei Vaccinfieber des Kaninchens nachgewiesen. Tanin o u. Y ta $^{5}$ haben bemerkt, dass die Veränderung der Blutmilchsäure bei Pleuritis in keinem Verhältnis zum Fieber steht. Fletcher u. Hopkins, ${ }^{6)}$ und Laquer ${ }^{7}$ betonen, dass sich die Muskelmilchsäure immer beträchtlich vermehrt, wenn der Muskel erwärmt wird.

Früher haben $\mathrm{K}$ a sai und $\mathrm{ich}^{8)}$ festgestellt, dass Milchsäure im ar. teriellen Blut des Kaninchens, das durch subkutane Injektion von Tetrahydro- $\beta$-Naphthylaminhydrochlorid oder Wärmestich in Fieberzustand gebracht ist, ausnahmslos zunimmt. Ob die Zunahme der Blutmilchsäure bei Fieber nun aber nur durch Spaltung des Leberglyko-

1) Fries, Bioch. Zeitschr., 1911, 35, 368.

2) Margreth, Folia clin., chim. et microsc., 1928, 3, 5; ref: in Kongr. Zentralbl. f. ges. inn. Med., 1928, 50, 564 .

3) Margreth, Boll. Soc. Ital. Biol. Sper., 1928, 3, 519.

4) Os a to, O ba u. K a sai, Chugai Iji Shimpo, 1926, 849.

5) Tanino u. Yata, Juzenkai Zasshi, 1928, 33, 2015.

6) Fletcher u. Hopkins, Journ. Physiol,, 1907, 35, 247.

7) La quer, H o p pe-Seyler's Zeitschr., 1921, 116, 169.

8) Adachi u, Kasai, wird bald in dieser Zeitschr. publiziert. 
gens verursacht wird oder ob sich die Zersetzung des Muskelglykogens auch daran beteiligt, um dies festzustellen, habe ich vorliegenden Versuch unternommen.

\section{Versuchsmethode.}

Als Versuchstiere wurde gesunde und möglichst wohlgenährte Hunde von etwa $20 \mathrm{~kg}$ Körpergewicht benutzt. Sie bekam am frühen Morgen im Hungerzustand subkutane Injektion 2\%iger Lösung von Morphinum hydrochloricum, $0,2 \mathrm{ccm}$ pro kg Körpergewicht, und etwa 30 Minuten danach, wenn sie in Narkose lagen, wurden sie auf dem elektrisch erwärmten Tierhaltergefesselt. Dann wurden nach dem Verzár, ${ }^{9}$ Barcroft u. Katoschen ${ }^{10)}$ Verfahren an der einen Hinterbein alle Gefässäste ausser einer Hauptarterie und Hauptvene, die in den und aus dem M. gastrocnemius führen, unterbunden, damit nur das durch diesen Muskel zirkulierende Blut zu untersuchen war. Zur Entnahme der Blutproben schob man eine Kanüle in die von der $V$. femoralis abzweigende $V$. saphena magna so hinein, dass das Blut aus der V. femoralis ohne die geringste Stauung entnommen werden konnte, und die andere Kanüle in den Nebenast der A. femoralis, um das arteriellen Blut zu entnehmen.

Als fiebererzeugendes Mittel diente ausschliesslich Tetrahydro- $\beta$ Naphthylaminhydrochlorid Merck (kurz: $\beta-T$ ), wovon subkutan eine 2 $\%$ ige wässerige Lösung $0,3 \mathrm{ccm}$ pro $\mathrm{kg}$ Körpergewicht injiziert wurde, bei einigen Fällen $0,35 \mathrm{ccm}$, stets im Hinblick auf den jeweiligen Ernährungszustand. Die Körpertemperatur wurde immer im Anus gemessen. Zur Bestimmung der Blutgeschwindigkeit wurden die Sekunden, die der Ausfluss von 1,0 ccm Venenblut durch die Pipette braucht, gemessen und daraus die in 1 Minute durch den Muskel fliessende Blutmenge ausgerechnet. Aus der Differenz der mittelst Barcroftschen $^{11)}$ Blutgasapparates gemessenen Sauerstoffunsättigung zwischen dem arteriellen und venösen Blut, das durch den Muskel laufen, der Blutgeschwindigkeit und dem Gewicht des Muskels wurde die Sauerstoffmenge, die $1 \mathrm{~g}$ Muskel in 1 Minute verbraucht, berechnet. Die Blut-CO $\mathrm{CO}_{2}$ wurde mit der Van Slykeschen ${ }^{12}$ Methode, der Blutzuckergehalt mit der Hage dorn- u. Jensen schen ${ }^{13)}$ Methode und die Blutmilchsäure mit der von $\mathrm{H}$ ay as a ka u. Inawash rro $^{14)}$ modifizier-

9) Verzá ár, Journ. Physiol.. 1912, 44, 243.

10) Barcroft u. Ka to, Philosoph. Transact. Royal Soc. London, 1915, 207, 149.

11) B a r c r oft, The respiratory function of the blood, Cambridge 1914, 290.

12) Van Slyke, Journ. Biol. Chem., 1917, 30, 305.

13) Hagedorn u. Jensen, Bioch. Zeitschr., 1923, 135, 46.

14) Hayasaka u. Inawashiro, Tohoku Journ. Exp. Med., 1928, 12, 1, 
ten Anrep u. Canna nschen ${ }^{15)}$ Methode ermittelt. Für diese Bestimmungen wurde jedesmal etwa $3 \mathrm{ccm}$ arterielles Blut und ebenso viel venöses Blut gebraucht, d.h. $1 \mathrm{ccm}$ zur Bestimmung des $\mathrm{O}_{2}$-Verbrauchs, $1 \mathrm{ccm}$ zu der der Milchsäure, $0,5 \mathrm{ccm}$ zu der des $\mathrm{CO}_{2}$ und die übrige Menge zur Bestimmung des Blutzuckers.

Kasai und ich $^{8)}$ hatten beim Kaninchen die Beeinflussung der Blutmilchsäuremenge infolge der Fesselung beobachtet und dabei festgestellt, dass die Blutmilchsäure 2 Stunden nach der Operation unter Fesselung wieder den Wert der Ruhe erreicht. Deshalb liess ich auch beim vorliegenden Versuch die Versuchstiere nach der Operation 2 Stunden lang auf dem Tierhalter ruhig liegen und entnahm ihnen erst dann das Blut, das zu den obigen Untersuchungen vor dem Fieber gebraucht wurde. Das arterielle und das vonöse Blut wurde jedesmal möglichst gleichzeitig entnommen. Nach subkutaner Injektion von $\beta$-T wurde die Körpertemperatur wiederholt gemessen und beim Stand des höchsten Fiebers zum zweiten Male Blut und danach für obige Untersuchungen stündlich 3 Stunden lang entnommen.

\section{Versuchsergebnisse.}

Versuch 1.

Hund, 古, 15,3 kg. Körpertemperatur $38,0 \mathrm{C}^{\circ}$. 20. XI. $1929.7^{\mathrm{h}}$ vorm. $3 \mathrm{ccm}$ $2 \%$ iger Morphinlösuug subkutan. $7 \mathrm{~h} 30^{\prime}$ vorm. Beginn der Operation, $8 \mathrm{~h} 40^{\prime}$ vorm. Ende der Operation. $11^{\mathrm{b}}$ vorm. $5,5 \mathrm{ccm} 2 \%$ iger $\beta$-T.-Lösung subkutan. Muskelgewicht $58 \mathrm{~g}$.

Tabelle 1.

(A : arterielles Blut, $\mathrm{V}$ : venöses Blut)

\begin{tabular}{|c|c|c|c|c|c|c|c|c|c|c|c|c|c|c|}
\hline \multirow[b]{2}{*}{$\begin{array}{c}\text { Zeit der } \\
\text { Blutentnahme }\end{array}$} & \multirow{2}{*}{ 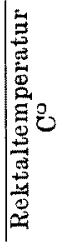 } & \multicolumn{2}{|c|}{$\begin{array}{l}\mathrm{O}_{2} \text {-Ver- } \\
\text { brauch }\end{array}$} & \multicolumn{2}{|c|}{$\begin{array}{l}\text { Blut- } \\
\text { strom }\end{array}$} & \multicolumn{3}{|c|}{$\begin{array}{c}\text { Blutzucker } \\
\text { (mg/dl) }\end{array}$} & \multicolumn{3}{|c|}{$\begin{array}{c}\text { Blutmilchsäure } \\
\text { (mg/dl) }\end{array}$} & \multicolumn{3}{|c|}{$\begin{array}{c}\text { Blut-CO }{ }_{2} \\
(\%)\end{array}$} \\
\hline & & 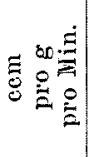 & 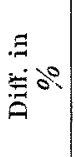 & 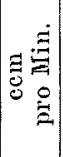 & 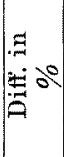 & A & $\mathrm{v}$ & $\left|\begin{array}{c}8 \\
x \\
x \\
4 \\
\mid 1 \\
|>|\end{array}\right|$ & A & $\mathrm{V}$ & $\left|\begin{array}{c}8 \\
\frac{8}{x} \\
4 \\
1 \\
|>|\end{array}\right|$ & A & $\mathrm{V}$ & $\mid \begin{array}{l}8 \\
\bar{x} \\
x \\
4 \\
1 \\
\mid-4\end{array}$ \\
\hline $\begin{array}{c}\text { Vor der } \beta-T- \\
\text { Injektion } \\
\text { Nach } 4 \text { St. } 30^{\prime} \\
\Rightarrow \quad 5 \text { St. } 30^{\prime} \\
\Rightarrow \quad 6 \text { St. } 30^{\prime} \\
\Rightarrow \quad 7 \text { St. } 30^{\prime}\end{array}$ & $\begin{array}{l}36,8 \\
39,5 \\
39,3 \\
39,1 \\
38,7\end{array}$ & $\begin{array}{l}0,0180 \\
0,0627 \\
0,0569 \\
0,0459 \\
0,0296\end{array}$ & $\begin{array}{r} \\
+248 \\
+216 \\
+155 \\
+\quad 64\end{array}$ & $\begin{array}{l}23 \\
32 \\
25 \\
21 \\
16\end{array}$ & $\begin{array}{r} \\
+39 \\
+\quad 9 \\
-\quad 9 \\
-30\end{array}$ & $\begin{array}{r}125 \\
88 \\
84 \\
88 \\
81\end{array}$ & $\begin{array}{r}122 \\
77 \\
74 \\
79 \\
74\end{array}$ & $\begin{array}{r}-2 \\
-13 \\
-12 \\
-10 \\
-9\end{array}$ & $\begin{array}{l}14,79 \\
20,57 \\
16,71 \\
14,79 \\
13,51\end{array}$ & $\begin{array}{l}19,94 \\
21,86 \\
18,00 \\
14,79 \\
13,51\end{array}$ & $\begin{array}{l}4+35 \\
6+6 \\
0 \\
+\quad 8 \\
\pm 0 \\
\pm 0\end{array}$ & \begin{tabular}{|}
46,32 \\
44,39 \\
42,46 \\
44,39 \\
46,32
\end{tabular} & $\begin{array}{l}57,90 \\
52,11 \\
55,01 \\
55,97 \\
57,90\end{array}$ & $\begin{array}{l}+25 \\
+17 \\
+30 \\
+26 \\
+25\end{array}$ \\
\hline
\end{tabular}

Die Körpertemperatur erreichte $4 \frac{1}{2}$ Stunden nach sublutaner Injektion des $\beta$-T ihren Höhepunkt, d. h. einen Anstieg von $2,2^{\circ} \mathrm{C}$, danach sank sie langsam, nämlich nach 3 Stunden erst um nur $0,8^{\circ} \mathrm{C}$.

15) Anrep.u. Cannan, Journ. Physiol., 1923, 58. 244. 
Der $\mathrm{O}_{2}$-Verbrauch zeigte beim höchsten Fieber ziemlich grosse Zunahme, sank aber danach allmählich und blieb am Schluss des Versuchs noch etwas vermehrt. Die Blutgeschwindigkeit veränderte sich fast parallel zum $\mathrm{O}_{2}$-Verbrauch, war am grössten bei höchster Temperatur, sank jedoch nach einer Stunde wieder beinahe zum Ruhewert, nahm dann fortdauernd ab und zeigte am Schluss des Versuchs ziemliche Abnahme im Vergleich zum Anfangswert. DerBlutzucker verminderte sich beträchtlich, später schwankte aber nur wenig, indem die Blutzuckerkurven des arteriellen und venösen Bluts beim höchsten Fieber beträchtlich auseinander gingen, dann allmählich näherten sich, aber weiter voneinander entfernt hielten als bei Ruhe. Wenige Zunahme zeigte die Blutmilchsäure beim Erreichen des maximalen Temperaturpunktes, erreichte aber nach 1 Stunde den Ruhewert und nahm danach allmählich ab. Die Milchsäurekurven des arteriellen und venösen Bluts näherten sich schon beim höchsten Fieber beträchtlich, danach immer mehr, um sich nach 2 Stunden zu vereinigen. Ad minimum vermindert sich die $\mathrm{Blut}^{-\mathrm{CO}_{2}}$ bei der grössten Temperatursteigerung, nahm danach immer mehr zu und erreichte am Schluss des Versuchs wieder den Anfangswert der Ruhe. Die $\mathrm{CO}_{2}$-Kurven des arteriellen und venösen Bluts näherten sich ein wenig beim höchsten Fieber, nach 1 Stunde aber entfernten sie sich, bis sie den Zustand der Ruhe erreichten.

Versuch 2.

Hund, 3, $26 \mathrm{~kg}$. Körpertemperatur $37,5 \mathrm{C}^{\circ}$. 13. XII. 1929. 6h $30^{\prime} 5,0 \mathrm{ccm}$ $2 \%$ iger Morphinlösung subkutan. $7^{\mathrm{h}}$ vorm. Beginn der Operation, $9^{\mathrm{h}}$ vorm. Ende der Operation. $11^{\mathrm{h}}$ vorm. 7,5 ccm $2 \%$ iger $\beta$-T.-Lösung subkutan. Muskelgewicht $84 \mathrm{~g}$.

Tabelle 2.

(A : arterielles Blut, V: venöses Blut)

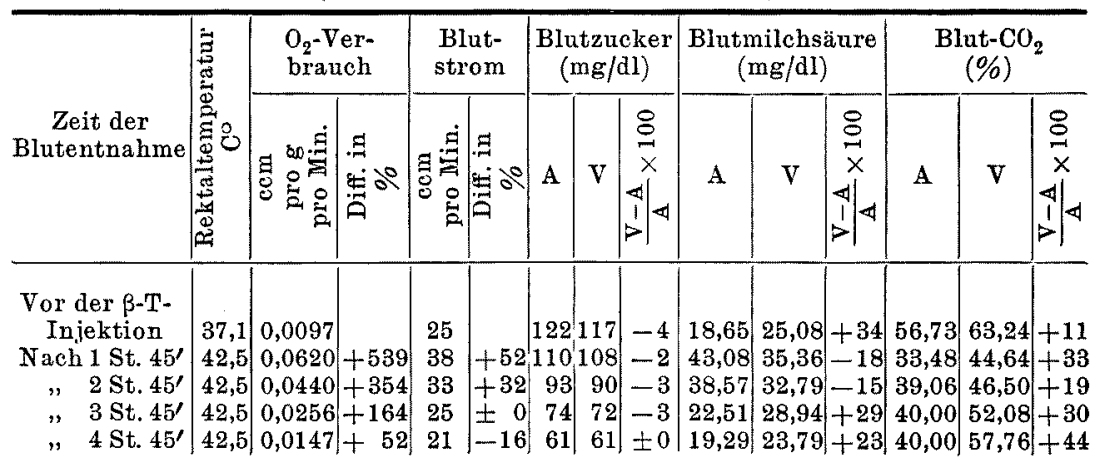

Bei diesem Versuch steigerte sich die Körpertemperatur plötzlich nach der Injektion von $\beta$-T nach $1 \frac{3}{4}$ Stunden um $5,4^{\circ} \mathrm{C}$, was dann während des Versuchs anhielt. Der $\mathrm{O}_{2}$-Verbrauch und die Blutgeschwindigkeit veränderten sich parallel zueinander wie beim Versuch 1, aber ihre Zunahme war beim Erreichen des höchsten Fiebers noch weit grösser, der erste war noch 3 Stunden danach ein wenig vermehrt, und die letzte gelangte nach 2 Stunden zum Ruhewert, um nach 3 Stunden noch darunter zu sinken. Der Blutzuckerspiegel erniedrigte sich beim grössten Temperaturanstieg anfangs ein wenig, später aber beträchtlich. Die Blutzuckerkurven des arteriellen und venösen Bluts, nachdem sie sich 
naherten, verliefen sich parallel zueinander und vereinigten sich nach 3 Stunden. Es zeigte sich meistens eine Zunahme der Blutmilchsäure bei der Fiebersteigerung, dauerte aber nicht lange, der Milchsäurespiegel kehrte nach 3 Stunden beinahe zum Ruhewert zurück. Der Milchsäuregehalt des arteriellen Bluts beim Erreichen des höchsten Fiebers bzw. 1 Stunde danach übertraf den des venösen Bluts beträchtlich, so dass so sich beide Kurven kreuzten,2 Stunden danach aber verminderte sich der erste schnell und erreichte wieder den Ruhewert. Die Blut- $\mathrm{CO}_{2}$ verminderte sich aufs Minimum anfangs, dann nahm allmählich zu, gelangte aber selbst nach 3 Stunden noch nicht wieder zum Ruhewert. Nach dem Erreichen des höchsten Fiebers divergierten die $\mathrm{CO}_{2}$-Kurven des arteriellen und venösen Bluts beträchtlich voneinander, nach 1 Stunde konvergierten sie jedoch einwenig, dann wieder allmählich divergierten sie, indem nach 3 Stunden die Entfernung voneinander am grössten war.

Versuch 3. (Fig. 1.)

Hund, 우, $25 \mathrm{~kg}$. Körpertemperatur $38,2^{\circ} \mathrm{C}$. 16. XII. 1929. $5 \mathrm{~h} 50^{\prime}$ vorm. 5 cem 2\%iger Morphinlösung subkutan. $6^{\mathrm{h}} 30^{\prime}$ vorm. Beginn der Operation, $8 \mathrm{~h} 20^{\prime}$ vorm. $7,5 \mathrm{ccm} 2 \%$ iger $\beta$-T.-Lösung subkutan. Muskelgewicht $93 \mathrm{~g}$.

Tabelle 3 .

(A: arterielles Blut, V: venöses Blut)

\begin{tabular}{|c|c|c|c|c|c|c|c|c|c|c|c|c|c|c|}
\hline \multirow[b]{2}{*}{$\begin{array}{c}\text { Zeit der } \\
\text { Blutentnahme }\end{array}$} & \multirow{2}{*}{ 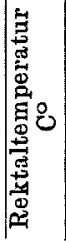 } & \multicolumn{2}{|c|}{$\begin{array}{l}\mathrm{O}_{2} \text {-Ver- } \\
\text { brauch }\end{array}$} & \multicolumn{2}{|c|}{$\begin{array}{l}\text { Blut- } \\
\text { strom }\end{array}$} & \multicolumn{3}{|c|}{$\begin{array}{c}\text { Blutzucker } \\
\text { (mg/dl) }\end{array}$} & \multicolumn{3}{|c|}{$\begin{array}{l}\text { Blutmilchsäure } \\
(\mathrm{mg} / \mathrm{dl})\end{array}$} & \multicolumn{3}{|c|}{$\begin{array}{c}\text { Blut- } \mathrm{CO}_{2} \\
(\%)\end{array}$} \\
\hline & & 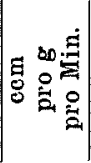 & 素 & 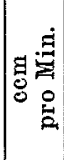 & $\begin{array}{l}a \\
00 \\
0\end{array}$ & $A$ & $\mathrm{~V}$ & $\begin{array}{c}8 \\
0 \\
x \\
4 \\
1 \\
1\end{array}$ & $A$ & V & $\left|\begin{array}{c|c|}8 \\
0 \\
x \\
4 \\
1 \\
|>| & 4\end{array}\right|$ & A & $\mathrm{V}$ & $\left|\begin{array}{c}8 \\
x \\
x \\
1 \\
1 \\
|>| d\end{array}\right|^{4}$ \\
\hline $\begin{array}{l}\text { Vor der } \beta \text {-T- } \\
\text { Injektion } \\
\text { Nach } 3 \text { St. } 30^{\prime} \\
\Rightarrow \quad 4 \text { St. } 30^{\prime} \\
" \quad 5 \text { St. } 30^{\prime} \\
" \quad 6 \text { St. } 30^{\prime}\end{array}$ & $\left|\begin{array}{l}38,0 \\
40,8 \\
40,9 \\
40,9 \\
40,7\end{array}\right|$ & $\left|\begin{array}{l}0,0101 \\
0,0498 \\
0,0285 \\
0,0160 \\
0,0095\end{array}\right|$ & $\begin{array}{r}+393 \\
+182 \\
+\quad 58 \\
-\quad 6\end{array}$ & $\begin{array}{l}12 \\
28 \\
18 \\
11 \\
10\end{array}$ & $\left|\begin{array}{rr}+ & 133 \\
+ & 50 \\
- & 8 \\
- & 17\end{array}\right|$ & \begin{tabular}{r|r}
97 & \\
92 & \\
102 & \\
92 & \\
97 &
\end{tabular} & $\begin{array}{l}88 \\
86 \\
95 \\
84 \\
90\end{array}$ & $\begin{array}{l}-9 \\
-7 \\
-7 \\
-9 \\
-7\end{array}$ & $\begin{array}{l}14,14 \\
26,40 \\
13,51 \\
13,51 \\
14,79\end{array}$ & $\begin{array}{l}18,00 \\
27,65 \\
16,71 \\
16,08 \\
16,71\end{array}$ & $\begin{array}{r}+27 \\
+\quad 5 \\
+24 \\
+19 \\
+13\end{array}$ & $\begin{array}{l}56,40 \\
40,42 \\
50,76 \\
52,64\end{array}$ & $\begin{array}{l}64,86 \\
48,88 \\
57,34 \\
60,16\end{array}$ & $\begin{array}{l}+ \\
+ \\
+\end{array}$ \\
\hline
\end{tabular}

Die höchste Körpertemperatur zeigte sich 3 Stunden 10 Minuten nach der $\beta$-T-Injektion, eine Steigerung um $2,9^{\circ} \mathrm{C}$ und schwankte dann nur wenig während des Versuchs. Der $\mathrm{O}_{2}$-Verbrauch und die Blutgeschwindigkeit veränderten sich ebenso wie bei Versuch 1 u. 2, der erste kam zum Anfangswert am Schluss des Versuchs ( $6 \frac{1}{2}$ Stunden nach der $\beta$-T-Injektion) zurück. Der Blutzucker nahm im Akme des Fiebers etwas ab, 1 Stunde danach einwenig zu, nach 2 Stunden nahm er wieder ab, aber am Schluss des Versuchs kam er beinahe auf den Anfangswert zurück. Die Kurven des arteriellen und venösen Blutzuckers näherten sich anfangs einwenig und hielten dann fast gleich weite Entfernung voneinander bis zum Schluss des Versuchs. Die Blutmilchsäure zeigte die grösste Vermehrung beim höchsten Fieber, aber kam schon nach 1 Stunde beinahe zum Ruhewert zurück. Der Unterschied zwischen des arteriellen und venösen Milchsäurespiegels verminderte sich bei der höchsten Fiebersteigerung, nahm danach einwenig zu, war aber dabei kleiner als im Ruhezustand. Im Akme des Fiebers nahm die Blut- $\mathrm{CO}_{2}$ erheblich ab, vermehrte sich dann all- 
Fig. 1. (Versuch 3.)

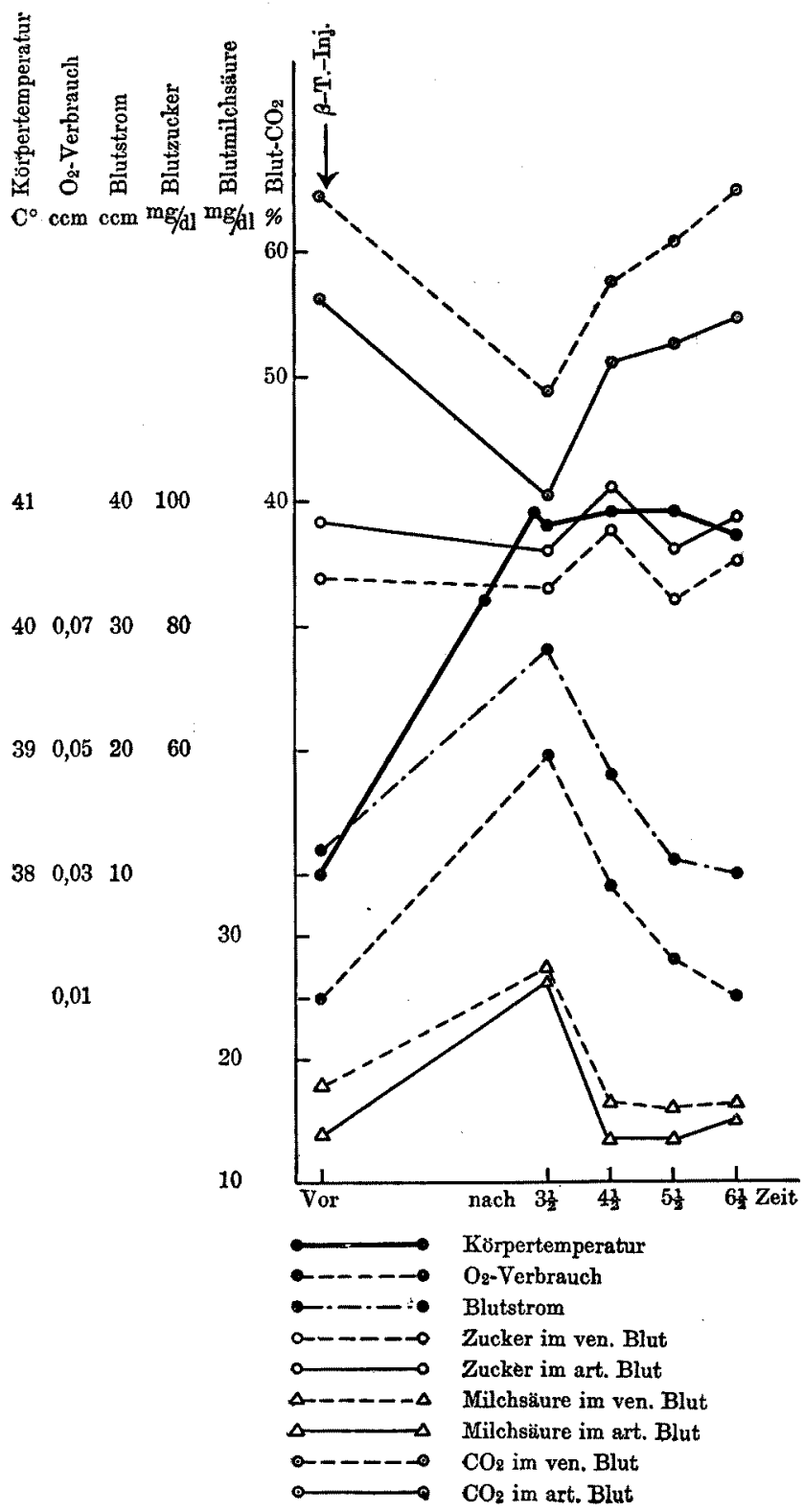


mählich und erreichte am Schluss des Versuchs wieder beinahe den Ruhewert. Die $\mathrm{CO}_{2}$-Kurven des arteriellen und venösen Bluts liefen im Anfang der Fiebersteigerung parallel zueinander, divergierten aber gegen Ende des Versuchs einwenig.

Versuch 4 .

Hund, o , $20 \mathrm{~kg}$. Körpertemperatur $38,0^{\circ}$ C. 7. I. $1930.5^{\mathrm{h}} 30^{\prime}$ vorm. $4,0 \mathrm{ccm}$ $2 \%$ iger Morphinlösung subkutan. $6 \mathrm{~h}$ vorm. Beginn der Operation, $7 \mathrm{~h} 30^{\prime}$ vorm. Ende der Operation. $9^{\mathrm{h}} 30^{\prime}$ vorm. 7,0 ccm $2 \%$ iger $\beta$-T.-Lösung subkutan. Muskelgewicht $73 \mathrm{~g}$.

$$
\text { Tabelle } 4 \text {. }
$$

(A: arterielles Blnt, V: venöses Blut)

\begin{tabular}{|c|c|c|c|c|c|c|c|c|c|c|c|c|c|c|}
\hline \multirow[b]{2}{*}{$\begin{array}{c}\text { Zeit der } \\
\text { Blutentnahme }\end{array}$} & \multirow{2}{*}{ 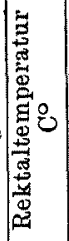 } & \multicolumn{2}{|c|}{$\begin{array}{l}\mathrm{O}_{2} \text {-Ver- } \\
\text { brauch }\end{array}$} & \multicolumn{2}{|c|}{$\begin{array}{l}\text { Blut- } \\
\text { strom }\end{array}$} & \multicolumn{3}{|c|}{$\begin{array}{c}\text { Blutzucker } \\
\text { (mg/dl) }\end{array}$} & \multicolumn{3}{|c|}{$\underset{(\mathrm{mg} / \mathrm{dl})}{\text { Blutmilchsäure }}$} & \multicolumn{3}{|c|}{$\begin{array}{c}\mathrm{Blut}-\mathrm{CO}_{2} \\
(\%)\end{array}$} \\
\hline & & 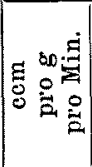 & $\begin{array}{l}\exists \\
400 \\
0.09\end{array}$ & 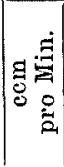 & E & A & $\mathrm{V}$ & $\left|\begin{array}{c}8 \\
\ddot{x} \\
4 \\
1 \\
1\end{array}\right|$ & $\mathbf{A}$ & $\mathrm{V}$ & $\left|\begin{array}{r}8 \\
8 \\
x \\
x \\
4 \\
1 \\
1 \\
1\end{array}\right|$ & A & V & $\left|\begin{array}{l}8 \\
\frac{8}{x} \\
1 \\
1 \\
1\end{array}\right|^{-4}$ \\
\hline $\begin{array}{l}\text { Vor der } \beta-T- \\
\text { Injektion } \\
\text { Nach } 3 \text { St. } \\
\Rightarrow \quad 4 \text { St. } \\
" \quad 5 \text { St. } \\
" \quad 6 \text { St. }\end{array}$ & $\begin{array}{l}37,5 \\
40,7 \\
40,9 \\
41,0 \\
41,0\end{array}$ & $\left\{\begin{array}{l}0,0214 \\
0,0601 \\
0,0487 \\
0,0381 \\
0,0288\end{array}\right.$ & $\begin{array}{r}+181 \\
+128 \\
+78 \\
+\quad 35\end{array}$ & \begin{tabular}{|l|}
17 \\
30 \\
25 \\
19 \\
18
\end{tabular} & $\begin{array}{r}+76 \\
+47 \\
+12 \\
+\quad 6\end{array}$ & \begin{tabular}{|r|}
104 \\
88 \\
84 \\
83 \\
88
\end{tabular} & $\mid \begin{array}{l}99 \\
86 \\
81 \\
70 \\
81\end{array}$ & $\begin{array}{r}-5 \\
-2 \\
-4 \\
-16 \\
-8\end{array}$ & \begin{tabular}{|l|l}
23,14 \\
36,65 \\
23,14 \\
6 & 25,08 \\
& 25,71
\end{tabular} & \begin{tabular}{|l}
30,22 \\
37,94 \\
31,51 \\
33,43 \\
33,43 \\
33,43
\end{tabular} & $\begin{array}{l}2 \\
4+31 \\
+4 \\
1 \\
+3 \\
+36 \\
3+33 \\
3+30\end{array}$ & $\mid \begin{array}{l}47,45 \\
35,11 \\
37,07 \\
37,07 \\
35,11\end{array}$ & $\begin{array}{l}53,1 \\
43.6 \\
47,4 \\
47,4 \\
47,4\end{array}$ & $\begin{array}{l}+12 \\
+24 \\
+28 \\
+28 \\
+35\end{array}$ \\
\hline
\end{tabular}

Die Körpertemperatur zeigte 2 Stunden 50 Minuten nach der Injektion von $\beta-T$ eine Steigerung von $3,2^{\circ} \mathrm{C}$, die auch danach noch anhielt.

Die Veränderungen des $\mathrm{O}_{2}$-Verbrauchs und der Blutgeschwindigkeit waren wie bei den vorigen Versuchen, ihre Anfangswerte kamen auch am Schluss des Versuchs (6 Stunden nach der Injektion) noch nicht zurück. Schon im Anfang des Fiebers zeigte sich eine erhebliche Abnahme des Blutzuckers, die weiter 2 Stunden lang fortdauerte. Der Blutzuckerspiegel zeigte am Schluss des Versuchs Neigung wieder herzustellen, lag aber noch immer niedriger als bei Ruhe. Die arterielle und venöse Blutzuckerkurveu konvergierten einwenig beim höchsten Fieber, divergierten dann aber allmählich, bis zum Maximum der Divergenz nach 2 Stunden, um dann am Schluss des Versuchs wieder zur Konvergenz zu neigen, wobei sie aber doch noch weiter voneinander entfernt waren als bei Ruhe. Die Blutmilchsäure vermehrte sich bei der grössten Fieberanfang und kam nach 1 Stunde beinahe auf den ursprünglichen Wert zurück. Die arterielle und die venöse Milchsäurekurve konvergierten einmal erheblich, kehrten aber nach 1 Stunde wieder zum Verhältnis bei Ruhe zurück. Während des Fiebers nahm die Blut- $\mathrm{CO}_{2}$ einmal beträchtlich ab, später aber etwas zu, blieb danach unverändert. Die $\mathrm{CO}_{2}$-Kurven des arteriellen und venösen Bluts divergierten beim höchsten Fieber einwenig und danach noch etwas stärker.

Versuch 5 .

Hund, $\delta, 23,5 \mathrm{~kg}$. Körpertemperatur $37,8^{\circ} \mathrm{C}$. 10. I. $1930.4^{\mathrm{h}} 30^{\prime}$ vorm. 4,8 ccm $2 \%$ iger Morphinlösung subkutan. $5^{\text {h }}$ vorm. Beginn der Operation, $7^{\mathrm{h}}$ vorm. Ende der Operation. $9^{\mathrm{h}}$ vorm. $8,2 \mathrm{ccm} 2 \%$ iger $\beta$-T.-Lösung subkutan. Muskelgewicht $95 \mathrm{~g}$. 
Tabelle 5 .

(A: arterielles Blut, V: venöses Blut)

\begin{tabular}{|c|c|c|c|c|c|c|c|c|c|c|c|c|c|c|}
\hline \multirow[b]{2}{*}{$\begin{array}{c}\text { Zeit der } \\
\text { Blutentnahme }\end{array}$} & \multirow{2}{*}{ 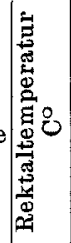 } & \multicolumn{2}{|c|}{$\begin{array}{l}\mathrm{O}_{2} \text {-Ver-- } \\
\text { brauch }\end{array}$} & \multicolumn{2}{|c|}{$\begin{array}{l}\text { Blut- } \\
\text { strom }\end{array}$} & \multicolumn{3}{|c|}{$\begin{array}{c}\text { Blutzucker } \\
(\mathrm{mg} / \mathrm{dl})\end{array}$} & \multicolumn{3}{|c|}{$\begin{array}{c}\text { Blutmilchsäure } \\
\text { (mg/dl) }\end{array}$} & \multicolumn{3}{|c|}{$\underset{(\%)}{\text { Blut-CO }}$} \\
\hline & & 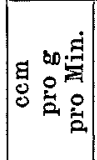 & 奋 & 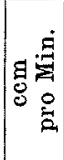 & 毒 & $\mathbf{A}$ & $\mathrm{v}$ & $\left|\begin{array}{c}8 \\
8 \\
x \\
4 \\
1 \\
1 \\
b\end{array}\right|$ & A & $\mathrm{V}$ & $\left|\begin{array}{c|}8 \\
8 \\
x \\
4 \\
1 \\
1 \\
p\end{array}\right|$ & $\mathbf{A}$ & $\mathrm{V}$ & $\left|\begin{array}{l}8 \\
x \\
x \\
1 \\
1 \\
\mid x\end{array}\right|$ \\
\hline $\begin{array}{l}\text { Vor der } \beta \text {-T- } \\
\text { Injektion } \\
\text { Nach } 4 \text { St. } 10^{\prime} \\
\Rightarrow \quad 5 \text { St. } 10^{\prime} \\
\Rightarrow \quad 6 \text { St. } 10^{\prime} \\
\Rightarrow 7 \text { St. } 10^{\prime}\end{array}$ & \begin{tabular}{|l|}
37,5 \\
41,9 \\
42,5 \\
42,2 \\
41,6
\end{tabular} & $\begin{array}{l}0,0120 \\
0,0262 \\
0,0393 \\
0,0230 \\
0,0163\end{array}$ & $\begin{array}{r}+118 \\
+228 \\
+\quad 92 \\
+\quad 36\end{array}$ & \begin{tabular}{|l|}
19 \\
25 \\
33 \\
30 \\
25
\end{tabular} & $\begin{array}{l}+32 \\
+74 \\
+58 \\
+32\end{array}$ & \begin{tabular}{|c|c|} 
& 111 \\
4 & 95 \\
8 & 86 \\
8 & 90 \\
2 & 83
\end{tabular} & $\begin{array}{r}104 \\
79 \\
75 \\
79 \\
74\end{array}$ & $\begin{array}{r}-6 \\
-17 \\
-13 \\
-12 \\
-11\end{array}$ & \begin{tabular}{|l|}
26,46 \\
44,46 \\
37,94 \\
34,08 \\
31,51
\end{tabular} & $\begin{array}{l}33,43 \\
47,58 \\
44,48 \\
39,28 \\
34,71\end{array}$ & $\begin{array}{l}+26 \\
+\quad 7 \\
+17 \\
+15 \\
+10\end{array}$ & \begin{tabular}{|l|}
47,20 \\
33,04 \\
32,10 \\
33,98 \\
36,86
\end{tabular} & $\begin{array}{l}55,6 \\
42,48 \\
43,4 \\
44,5 \\
45,35\end{array}$ & $\begin{array}{l}+18 \\
+29 \\
+29 \\
+35 \\
+31 \\
+23\end{array}$ \\
\hline
\end{tabular}

Bei diesem Fall erhöhte sich die Körpertemperatur 4 Stunden nach der $\beta$-T-Injektion schon ziemlich bedeutend und erreichte 5 Stunden danach ihren Höhepunkt, wobei sie eine Steigerung $\nabla$ on $5^{\circ} \mathrm{C}$ zeigte; danach sank sie allmählich. Der $\mathrm{O}_{2}$-Verbrauch und die Blutgeschwindigkeit zeigten schon vor dem Erreichen des höchsten Fiebers ziemlich erheblich Vermehrung, die beim Fieberakme das Maximum erreichte; danach nahmen sie allmählich ab, aber am Schluss des Versuchs (7 Stunden nach der Injektion) erholten sie doch noch nicht gänzlich. Die Abnahme des Blutzuckers ging Hand in Hand mit der Fiebersteigerung. Die Zuckerkurven des arteriellen und venösen Bluts divergierten beträchtlich, bevor die Temperatur das Maximum erreichte, näherten sich dann allmählich, aber waren am Schluss des Versuchs noch weiter voneinander entfernt als bei Ruhe. Die Blutmilchsäure vermehrte sich bis auf ihr Maximum früher als die Temperatur, nahm dann langsam ab und kam gegen Ende des Versuchs fast auf den ursprünglichen Wert. Die arterielle und die venöse Milchsäurekurve konvergierten schon vor dem Erreichen des höchsten Fiebers am nächsten und divergierten dann während 2 Stunden allmählich, um wieder am Schluss des Versuchs zu konvergieren.

Die Blut- $\mathrm{CO}_{2}$ zeigte im Anfang des Fiebers grosse Abnahme, vermehrte sich dann langsam, aber hatte gegen Ende des Versuchs noch nicht wieder den Ruhewert erreicht. Die arterielle und die venöse $\mathrm{CO}_{2}$-Kurve wiesen Divergenz auf, die beim höchsten Fieber am grössten war, danach aber konvergierten sie mit dem Sinken des Fiebers allmählich wieder.

\section{Zusammenfassung und Besprechung.}

Der Mechanismus der Körpertemperaturregulation ist mehr oder weniger verschieden je nach der Art des Tiers; beim Hunde beteiligt sich wesentlich die chemische Regulation daran. Diese bewirkt hauptsächlich Glykogen als Triebkraft, woraus verständlich wird, dass die Leber, die reichlich Glykogen enthält, bei der Wärmebildung grosse Rolle spielt; bei einem Tier mit glykogenarmer Leber tritt keine Stei- 
gerung der Körpertemperatur ein, wenn man verschiedene fiebererzeugende Mittel darauf einwirken lässt; auch bei Tieren, die infolge Hungers kein Leberglykogen haben, ruft Wärmestich wie Injektion fiebererzeugender Mittel kein oder nur leichtes Fieber hervor.

Nach der Angabe von Hirsch u. Mülle ${ }^{16}$ ) hat die Leber immer höhere Wärme als die anderen Organe. Manasse ${ }^{1}{ }^{17}$ ) $\mathrm{May}^{18}$ ) $\mathrm{u}$.a. fanden, dass das Leberglykogen des Kaninchens beim Fieber beträchtlich abnimmt; dasselbe haben Dadlez u. Koskowski ${ }^{19}$ ) beim Methylenblaufieber bestätigt. Die in der Leber lokalisierte chemische Wärmeregulation wird nach $\mathrm{Plaut}{ }^{20)}$ durch Zerstörung des Plexus hepaticus fortfallen.

Eine Reihe der Autoren sind der Ansicht, dass das Muskelglykogen eigentlich der Ursprung der Energien bei der Muskelarbeit ist und sich kaum an der Fieberbildung beteiligt.

Hirsch u. Rolly $\mathbf{y}^{21}$ betonen, dass selbst wenn die Nervenendigungen im quergestreiften Muskel durch Curare gelähmt werden, Wärmestich oder ein fiebererzeugendes Mittel im Stand ist, Fieber hervorzurufen, ganz wie beim intakten Tier. May, ${ }^{18}$ ) Dadlez u. Koskowski ${ }^{19}$ haben bei den oben erwähnten Fieberversuchen bemerkt, dass Muskelglykogen vielmehr zunimmt.

Bei dieser Sachlage ist es für die Erforschung der Wärmebildung wichtig, die Veränderungen der Abbauprodukte des Glykogens, d. h. des Blutzuckers, der Blutmilchsäure, der Blut- $\mathrm{CO}_{2}$ u. a. im Muskel bei gesteigerter Temperatur zu beobachten, um den intermediären Stoffwechsel des Muskelglykogens bei Fieber zu untersuchen.

Auf Grund meiner Versuchsergebnisse betrachte ich zunächst den Sauerstoffverbrauch des Muskels bei Fieber:

Bei Fieber zeigt er immer grosse Zunahme, beim Versuch 2 vermehrte er sich um mehr als $500 \%$, was auf eine beträchtliche Steigerung des Stoffwechsels im Muskel bei starkem Fieber hinweist; später nimmt der Sauerstoff verbrauch ziemlich ab, wenn auch das Fieber sich fast auf derselben Höhe hält, er bleibt jedoch noch mehr oder weniger vergrössert als der ursprüngliche Wert. Im Einklang mit der Ansicht von Dürr, $\left.{ }^{22}\right)$ Ito, ${ }^{23}$ Ha yasaka, ${ }^{24}$ Kodera u. Adachi ${ }^{25)}$ kann der $\mathrm{O}_{2}-$ Verbrauch hier mit Recht abnehmen, weil, wie Akiy a ${ }^{967}$ neulich festgestellt hat, im Stadium des steigenden Fiebers Alkalosis und doch mit der Fieberdauer stärkere Acidosis eintritt.

16) Hirseh u. Müller, Dtsch. Arch. f. klin. Med., 1903, 75, 286.

17) Manassein, Virchows Archiv, 1872, 56, 220.

18) M a y, Zeitschr. f. Biol., 1894, 30, 1.

19) Dadlez u. Koskowski, C.R. Soc. Biol., 1927, 96, 576.

20) Pla ut, Zeitschr. f. Biol, 1922, 76, 183.

21) Hirsch u. Rolly, Dtsch. Arch. f. kl. Med., 1903, 75, 307.

22) D ürr, Zeitschr. f. ges. exp. Med., 1925, 46, 573 .

23) Ito, Tohoku Jonrn. Exp. Med, 1926-27, 8, 75.

24) Hayas aka, Tohoku Journ. Exp. Med.. 1930, 14, 487.

25) Kodera u. Ad achi, wird bald in dieser Zeitschr. publiziert.

26) Akiy a, Zeitschr. f. klin. Med., 1928, 109, 312. 
Die Blutges ch windigkeit vermehrt sich im Anfangsstadium des Fiebers parallel zum Sauerstoffverbrauch, was mit der Ansicht von Verzári ${ }^{27}$ und Ich imi i ${ }^{28)}$ übereinstimmt. Und obgleich das bei diesem Versuch benutzte $\beta$-T ein Mittel ist, den Sympathikus zu reizen und periphere Blutgefässe zu kontrahieren, wird doch die Blutströmung sehr beträchtlich vergrössert; dies kommt wohl daher, dass bei Fieber der Stoffwechsel des Muskels so lebhaft wird, dass er Lieferung einer grossen Menge $\mathrm{O}_{2}$ erfordert. Wie der Sauerstoffverbrauch wendet sich auch der Blutstrom frühzeitig zur Verminderung trotzdem die gesteigerte Körpertemperatur sich in gleicher Höhe hält; das dürfte auf die dabei eingetretene hochgradige Azidose zurückzuführen sein. Nach der Ansicht von Fleisch, ${ }^{29)}$ Atzler, u. Lehmann, ${ }^{30)}$ Hess,${ }^{31)}$ Hayasaka, ${ }^{25)}$ Kodera u. A dachi ${ }^{25}$ müssten sich bei stärkerer Azidose die peripheren Blutgefässe kontrahieren und zur Abnahme der Blutströmung führen.

Blutzucker: Betreffs der Veränderung des Blutzuckerspiegels bei Fieber gibt es viel Untersuchungen.

Seitdem Liefmann u. Stern ${ }^{32}$ ) Zunahme des Blutzuckergehaltes bei fiebernden Kranken beobachtet haben, haben Hollinger, ${ }^{33}$ Freund u. Marchand, ${ }^{34)}$ Rolly u. Oppermann, ${ }^{35}$ ) Furonaka ${ }^{36}$ u. a. klinisch dasselbe nachgewiesen, und das gleiche ergab sich bei dem Tierversuch von $\mathrm{Cohn},{ }^{37}$ Morita u. $\mathrm{Naito}^{38}$ ) äusserten sich dagegen, dass beim Fieber keine Zunahme des Blutzuckers eintrete, und Geig er ${ }^{39}$ dass der Blutzuckerspiegel bei Fiebersteigerung zwar steigt, zur Zeit des voll entwickelten Fiebers jedoch im $\mathrm{Ge}-$ genteil sinkt.

Bekanntlich steht der Stoffwechsel bei Fieber in enger Beziehung zu den Drüsen der inneren Sekretion. Euler ${ }^{40}$ wies Hyperadrenalinämie beim fiebernden Tier und $\mathrm{Sug}$ a wara $\mathrm{a}^{41}$ eine geringe Zunahme der Adrenalinsekretion bei Injektion von Naphthylamin nach.

Masin ${ }^{42}$ berichtet, dass sich infolge der Zersetzung des Leberglykogens durch Adrenalininjektion der Blutzuckerspiegel steigt, was

27) Verzár, Pflü ge r's Arch., 1920, 183, 239.

28) I c hi mi, Tohoku Journ. Exp. Med., 1929. 13, 100.

29) Fleis ch, Pfllüger's Arch., 1918, 171, 86.

30) Atzler u. Lehmann, Pflüger's Arch., 1921, 190, 118.

31) Hess, Ergebn. d. inn. Med. u. Kinderheilk., 1923, 23, 1.

32) Liefmann u. Stern, Bioch. Zeitschr., 1906, 1, 299.

33) Hollinger, Dtsch. Arch. f. klin. Med., 1908, 92, 217.

34) Freund u. Marchand, Dtsch. Arch. f. klin. Med., 1913, 110, 120.

35) Rolly u. Oppermann, Bioch. Zeitschr., 1913, 48, 50.

36) Furonak a, Chosen Igakkai Zasshi, 1930, 20, 147.

37) Cohn, Zeitschr. f. ges. exp. Med., 1927, 53, 878.

38) Morita, u. Na ito, Tohoku Journ. Exp. Med., 1921, 2, 562.

39) Geiger, Arch. f. exp. Pathol. u. Pharmakol., 1927, 121, 67.

40) Euler, Pflüger's Arch., 1927, 217, 699.

41) Sugawara, Tohoku Journ. Exp. Med., 1927, 9, 368; Sato u. Sugawara Ibid., 1930, 16, 580 .

42) Masing, Arch. f, exp. Pathol. u. Pharmakol., 1912, 69, 431. 
aber nach 15-20 Minuten infolge des Eintritts der Oxydationshemmung aufhört.

Wenn also bei Fieber die Adrenalinsekretion beschleunigt wird, so müsste hier Adrenalinhyperglykämie eintreten. In der Mehrzahl meiner Versuche aber sank der Blutzuckerspiegel schon beim Erreichen des höchsten Fiebers, er wurde mit der Fieberdauer immer mehr erniedrigt. Diese Verminderung des Blutzuckers kann darauf zurückgeführt werden, dass das Leberglykogen bei höherem Fieber in grösserem Masse zu Milchsäure gespaltet wird, vielleicht obschon, wie in Ichim is ${ }^{28)}$ Ergebnis, die Adrenalinsekretion vor der Erreichung des höchsten Fiebers zunimmt und zur Hyperglykämie führen dürfte.

Im Fieberakme divergierten die Blutzuckerkurven des im Muskel zirkulierenden arteriellen und venösen Bluts (Versuch $1 \mathrm{u}$. 5) infolge grösserer Abnahme des venösen Blutzuckergehalts, d.h. infolge des vermehrten Zuckerverbrauchs im Muskel, wie das Ich imi $i^{28)}$ schon bei gleichem Versuche bemerkte; bei einigen Fällen (Versuch 2, 3 u. 4) dagegen konvergierten sie, hier scheint also als ob der Zuckerverbruch im Muskel abnahm. Da aber in diesen Fällen der Blutstrom beschleunigt ist, so ist von der Zeiteinheit aus betrachtet, der in einer Minute im Muskel verbrauchte Blutzucker, wie es Versuch 3 (Fig. 2) deutlich zeigt, vielmehr vermehrt. Aus der Tatsache, dass bei hohem Fieber der Sauerstoff verbrauch des Muskels beträchtlich zunimmt, kann man offenbar auf gesteigerten Stoffwechsel im Muskel, der den vermehrten Verbrauch des Zuckers des Bluts im Muskel in Anspruch nimmt, schliessen.

Später nimmt der Zuckerverbrauch des Muskels bei Fieberdauer allmählich ab, was durch Auftreten starker Azidose bedingt ist. Dasselbe haben auch Kodera u. $\mathrm{ich}^{25)}$ am Hunde beim Erzeugen künstlicher Azidose durch Salzsäureinjektion beobachtet.

Blutmilchsäure: Bei jedem Versuchfalle beobachtet man Zunahme der Milchsäure sowohl im arteriellen als auch im venösen Blut, wenn das Fieber beträchtlich steigt. Wie aus den Versuchen von May, ${ }^{18}$ Dadlez u. Koskow ski ${ }^{19}$ erhellt, bei Fieber nimmt das Leberglykogen ab. Die gesteigerte Adrenalinsekretion, die infolge der Temperatursteigerung durch $\beta$-T-Injektion hervorgerufen ist, fördert, wie Kimura u. Ka to ${ }^{43}$ festgestellt haben, die Glykogenolyse in der Leber und führt die Vermehrung der Blutmilchsäure herbei.

Bald nimmt aber die Blutmilchsäure trotz des davernden Fiebers und kehrt nach 1-2 Stunden zum ursprünglichen Wert zurück, was daher kommen dürfte, dass mit dem Fieber nicht nur Azidose eintritt und die Glykogenolyse gehemmt wird, sondern auch, wie Liljestrand $u$.

43) K i mura u. Ka to, wird bald in dieser Zeitschr. publiziert. 
Wils $\mathrm{n}^{44)}$ u. a. feststellten, die Michsäure im Blut teilwieise in den Harn vermehrt ausgeschieden wird.

Bemerkenswert ist hier aber das Verhältnis zwischen den Veränderungen des Milchsäurespiegels im arteriellen und des im venösen Blut bei Fieber; im Anfangstadium des Fiebers geht die Zunahme der venösen Blutmilchsäure im Muskel nicht parallel zu der der arteriellen, indem die erste sich weniger als die letzte vermehrt, so dass die beiden Kurven sich zueinander nähern, was darauf hinweist, dass die Milchsäuremenge in dem in einer Zeiteinheit durch den Muskel laufenden Blut abnimmt. Diese Abnahme hat ihre Ursache entweder in verminderter Milchsäurebildung aus dem Muskelglykogen oder gesteigerter Milchsäureresynthese im Muskel bei Fieber.

Fig. 2. (Versuch 3.)

Kurven des Zuckerverbrauches, der Milchsäure- u. $\mathrm{CO}_{2}$-Bildung im M. gastrocnemius in einer Minute.

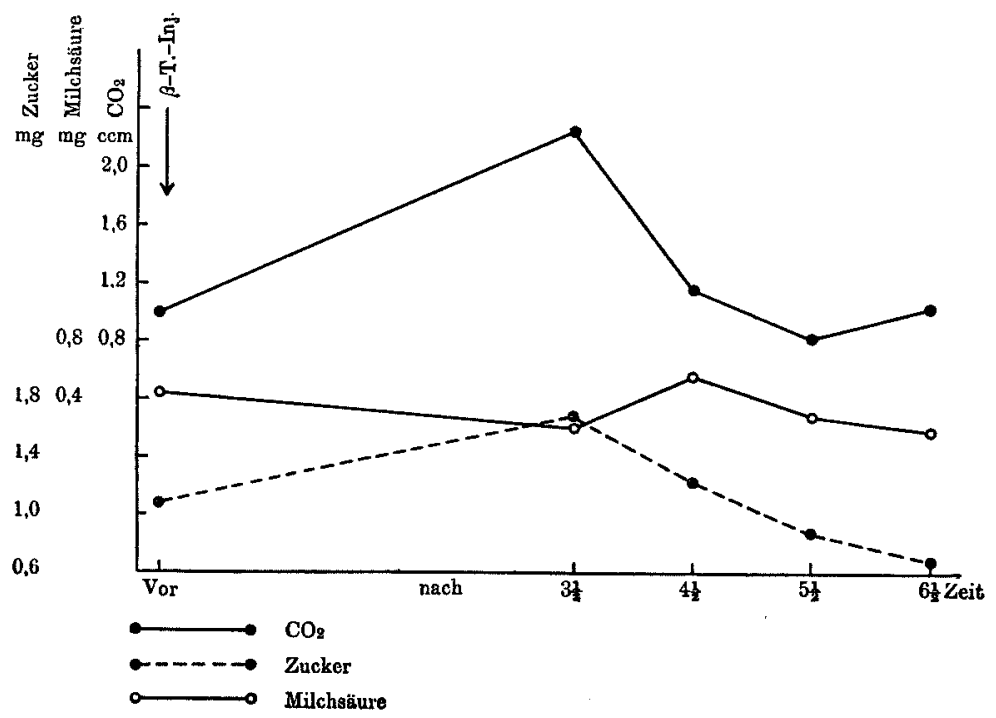

Aus dem Vergleich der Zucker-, der Milchsäure- und der $\mathrm{CO}_{2^{-}}$ Kurven des in einer Zeiteinheit durch den Muskel laufenden Bluts untereinander ersieht man nun, dass mit der Abnahme der Milchsäurebildung im Muskel die $\mathrm{CO}_{2}$ Bildung in ihm meistens zunimmt, dass sich auch der Zuckerverbrauch des Muskels vermehrt, oder falls dieser abnimmt, die Abnahme viel geringer ist als die der Milchsäurebildung.

44) Liljestrand u. Wils on, Journ. Biol. Chem., 1925, 65, 773. 
Daraus dürfte man schliessen, dass im Anfang des Fiebers die Resynthese der im Muskel gebildeten Milchsäure lebhaft vor sich geht, wobei diese teilweise zu Lactacidogen wieder gebaut wird, und doch erfolgt andererseits die Verbrenrıung der Milchsäure unter üppiger Sauerstofflieferung und beschleunigter Blutströmung gut, indem sie in $\mathrm{CO}_{2}$ und $\mathrm{H}_{2} \mathrm{O}$ zersetzt wird, wodurch sich die Milchsäure in Muskelblut beträchtlich vermindert. Dass bei beträchtlicher Zunahme der Milchsäure im arteriellen Blut ihre Resynthese in Skelettmuskel sehr lebhaft vor sich geht, beobachtete $\mathrm{H}$ a y a s a $\mathrm{ka}^{45)}$ an Beriberikranken nach Arbeit.

Nach Kondo, ${ }^{46)}$ Kodera u. Adachi ${ }^{25)}$ wird die Milchsäurebildung im Muskel durch Säure gehemmt, und nach $\mathrm{May}^{18}$ ) soll das Leberglykogen nach Fieber beträßhtlich abnehmen oder sogar verschwinden, hingegen sich das Muskelglykogen vermehren. Aus diesen Angaben ist es doch nicht auszuschliessen, dass im Anfang des Fiebers im Muskel die Milchsäurebildung aus Glykogen gehemmt wird.

Die Milchsäurebildung im Muskel, die im Anfang des Fiebers verminderthat, nimmt trotz der Dauer des Fiebers einmal wieder zu mit der Abnahme der Blutgeschwindigkeit und des $\mathrm{O}_{2}$-Verbrauchs, die Resynthese und Zersetzung der Milchsäure im Muskel wird vermindert. Das ist auch zu ersehen nicht nur aus dem Verlauf der Kurven der Milchsäurebildung im Muskel in einer Zeiteinheit, sondern auch daraus, dass die Milchsäurekurven des venösen und arteriellen Bluts eine Neigung zur Divergenz haben, indem die Kurve des ersten langsamer sinkt als die des letzten. Diese Zunahme der Milchsäurebildung hört bald auf, die Milchsäurebildung nimmt wieder ab.

Blut-CO : Dass bei Fieber die Blut- $\mathrm{CO}_{2}$ abnimmt, ist schon von $\mathrm{Haggard}{ }^{47} \mathrm{Kuhlmann},{ }^{48)} \mathrm{Beck}^{499} \mathrm{Akiya}^{26)}{ }^{26}$ Bühl u. Heinz ${ }^{50\rangle}$ u. a. nachgewiesen. Bei meinem Versuch vermindert sich die Blut$\mathrm{CO}_{2}$ beträchtlich mit Fieberanfang, dann zeigt sie die Neigung, allmählich wieder auf den ursprünglichen Wert zu steigen, trotzdem sich das Fieber fast auf derselben Höhe hält. Der Unterschied der $\mathrm{CO}_{2}$-Menge zwischen dem im Muskel zirkulierenden arteriellen und dem venösen Blut nimmt bei Fieber im allgemeinen zu infolge der Abnahme der Verminderung des letzten; das rührt wohl daraus her, dass dabei die oxydative Stoffzersetzung im Muskel lebhaft wird; dies wird durch die beträchtliche Zunahme des $\mathrm{O}_{2}$-Verbrauchs im Muskel bewiesen.

45) Hayasaka, Tohoku Journ. Exp. Med., 1929, 14, 85.

46) Kond o, Bioch. Zeitschr., 1912, 45, 63.

47) Haggard, Journ. Biol. Chem., 1920, 44, 131.

48) Ku hlma n n, Dtsch. Arch. f. klin. Med., $1920,33,346$.

49) - Beck, Jahrb. f. Kinderheilk, 1926, 113, 198.

50) Brühl u. Heinz, Zeitschr. f. ges. exp. Med., 1928, 62, 525. 


\section{Schluss.}

Aus der zeitlichen Beobachtung des Gasstoffwechsels, des Kohlehydratstoffwechsels und des Blutkreislaufszustands im Muskel des infolge der Injektion von Tetrahydro- $\beta$-Naphthylmain fiebernden Hundes kann folgender Schluss gezogen werden:

1. Der $\mathrm{O}_{2}-$ Verbrauch des Muskels vermehrt sich mit dem Fieber und vermindert sich dann verhältnismässig schnell, trotzdem sich das Fieber auf demselben erhöhten Grad hält, gelangt aber selten zum normalen Wert zurück.

2. Die Blutgeschwindigkeit verändert sich fast parallel zum $\mathrm{O}_{2}-$ Verbrauch.

3. Der Blutzucker vermindert sich meistens im Anfangstadium des Fiebers und hat dann auch die Neigung, mit der Fieberdauer immer mehr abzunehmen.

4. Der Unterschied im Zuckergehalt zwischen dem arteriellen und dem venösen Blut des Muskels scheint zuweilen abzunehmen, aber nach der Blutstromgeschwindigkeit berechnet, vermehrt sich der Zukkerverbrauch im Muskel in einer Zeiteinheit meistens.

5. Die Blutmilchsäure im Muskel vermehrt sich beim höchsten Fieber beträchtlich und vermindert sich dann schnell, trotzdem sich das Fieber fast auf derselben Höhe hält, und kommt bald auf den ursprünglichen Wert zurück.

6. Die Milchsäurebildung im Muskel verminder't sich bei Fieber. Bei höherem Fieber beträgt der Milchsäuregehalt in dem aus dem Muskel ausfliessenden venösen Blut weniger als in dem in ihn einströmenden arteriellen Blut; das dürfte hauptsächlich daher kommen, dass die Zersetzung wie die Resynthese der Milchsäure im Muskel gefördert wird. Später wird aber die Milchsäure des aus dem Muskel ausfliessenden Bluts reaktionär einmal grösser als die des arteriellen Bluts; was auf den Rückgang des oben erwähnten Mechanismus des Milchsäurestoffwechsels hinweist.

7. Die Blut- $\mathrm{CO}_{2}$ vermindert sich beim höchsten Fieber beträchtlich und neigt dann dazu, allmählich auf den normalen Wert zurückzukommen.

8. Bei Fieber wird die $\mathrm{CO}_{2}$-Bildung im Muskel beschleunigt. 